\title{
LA URGENTE NECESIDAD DE PENSAR OPERACIONALMENTE
}

\section{RESUMEN}

Mayo E.M.C. Christian Gniesko ${ }^{1}$

Existieron hechos recientes en américa latina que pusieron en la vidriera nuevamente a las Fuerzas Armadas, teniendo que actuar en operaciones sumamente delicadas, donde el riesgo era muy alto y el margen de error casi nulo.

Observamos que las técnicas y procedimientos utilizadas no eran adecuadas para la administración de la crisis, junto a ello, el manejo de la información en redes sociales jugó un papel trascendente en su actuar. Se debe sumar, además, que la estructura conceptual de "toma de decisiones" utilizada, se basan en procedimientos de la pos $2 \mathrm{da}$ guerra mundial, totalmente atemporales.

Por ello, el presente trabajo abordará una forma de pensar distinta a la actual, para ser usada en este tipo de crisis, trabajando de manera innovadora para poder presentar soluciones adecuadas con esquemas de toma de decisiones más acordes a los tiempos que se viven.

Comenzará con la necesidad de pensar en las relaciones de los distintos niveles de la conducción en el manejo de las crisis, reflexionando sobre las responsabilidades e insumos a producir para cada uno de ellos.

Luego nos describirá brevemente qué es el "arte y diseño operacional", su empleo y virtudes para ser adoptado por las Fuerzas Armadas del Ecuador, finalizando con algunas propuestas necesarias para dotar a la organización de mayor eficiencia y actualización al estado del arte sobre la temática.

Palabras claves: Crisis - Diseño - Operacional - Complejidad - Creatividad.

\begin{abstract}
There were recent events in Latin America that put the Armed Forces in the window again, having to act in extremely delicate operations, where the risk was very high and the margin of error almost zero.

We observe that the techniques and procedures used were not suitable for the administration of the crisis, together with this, the management of information on social networks played a transcendent role in their actions. It should also be added that the conceptual structure of "decision-making" used, are based on procedures of the 2nd world war, totally timeless.
\end{abstract}

Therefore, this work will address a way of thinking different from the current one, to be used in this type of crisis, working in an innovative way to be able to present adequate solutions with decision-making schemes more in line with the times that are lived.

It will begin with the need to think about the relationships of the different levels of driving in crisis management, reflecting on the responsibilities and inputs to be produced for each of them.

Then he will briefly describe what "art and operational design" is, its use and virtues to be adopted by the Armed Forces of Ecuador, ending with some proposals necessary to provide the organization with greater efficiency and updating the state of the art on the subject.

Keywords: Crisis - Design - Operational - Complexity - Creativity

\footnotetext{
1 gniesko@yahoo.com.ar

EJÉRCITO DE ARGENTINA
} 


\section{Introducción}

Los acontecimientos de octubre del 2019 en el Ecuador, Bolivia y Chile, sumados a otros hechos con similares connotaciones en otras partes del mundo, ponen sobre el tapete la actuación de las Fuerzas Armadas, a pesar que sus roles se encuentran perfectamente definidos en las Cartas Magnas o leyes de la mayoría de los Estados en el mundo, a la hora de actuar sus acciones no resultan ser tan nítidas como pareciera.

La pregunta es: ¿por qué sucede esta dicotomía entre lo normado y la realidad?, ante ella hay variadas respuestas desde distintas ópticas y diversos temas en cuestión, pero el presente escrito abordará sólo el plano técnico-conceptual que nos concierne, el planeamiento y la ejecución de las operaciones para cumplir con las órdenes del nivel estratégico nacional.

Debemos tener presente que, el empleo de las Fuerzas Armadas va complejizándose conforme se lo exige más y más al Estado-Nación, ésta entidad jurídica que encuentra crisis constantes en el plano económico, social, laboral, descontento de ciertas acciones o políticas implementadas, terremotos, inundaciones, incendios forestales, etc.; es decir que convive con la crisis en forma permanente.

En el entorno descripto, las Fuerzas Armadas como institución ligada a dar respuesta en dichas situaciones, a veces como responsables primarias $u$ otras en apoyo a instituciones, se verán inmiscuidas en este delicado ambiente, debiendo generar respuestas rápidas y eficientes para salir de la crisis, en la mayoría de los casos con personal y medios que muchas veces no se encuentran preparados ni equipados convenientemente para el empleo exigido.

Las Fuerzas Armadas tienen una gran responsabilidad junto con las autoridades políticas del Estado, pero intrínsecamente su actuar debe estar totalmente desprovisto de politización, así nos recuerda Exum (2017) que "... los ciudadanos deben confiar en que los oficiales militares nunca usarán sus armas para lograr un fin político...” (pág. 1). Deben contar en el plano técnico del planeamiento, con herramientas depuradas y acordes a los momentos que se vive, no se puede depender de procesos productores de órdenes que se utilizaban en la pos 2da Guerra Mundial, muchos de los cuales derivan en métodos de toma de decisiones que no tienen en cuenta todas las dimensiones operativas de las fuerzas. Y éste, es sin lugar a dudas un gran desafío que se deberá abordar ahora, no es un problema a resolver en el futuro.

\section{Desarrollo}

El responsable político de las crisis, es por naturaleza, el nivel estratégico nacional en cabeza del Poder Ejecutivo, junto a él su comité de crisis normado cons- titucionalmente para tal fin, para ello contará con organismos de las distintas áreas de responsabilidad del Estado, quienes asesorarán y asistirán conforme sus atribuciones (En el Ecuador se lo denomina COSEPE). Pese a estar claramente establecidas las actividades de los organismos o consejos, en variadas oportunidades que nos arrojan la historia y sucesos recientes, este nivel estratégico no presenta productos de análisis convenientemente conformados, para que puedan ser un insumo adecuado en el planeamiento del siguiente nivel de la conducción, que es el operacional u operativo, el que deberá traducir esas intenciones estratégicas en objetivos alcanzables. Existieron crisis donde los consejos no han actuado como asesores, se han tomado decisiones que no han tenido trabajo previo de asesoramiento inter-agencial, y otros tantos errores que doctrinariamente son inaceptables.

Esos errores comunes, que muchas veces se deben al abordaje con premura de situaciones de crisis, donde se toman decisiones erróneas debido a la necesidad de dar soluciones rápidas, que tampoco se piensa que desembocarán en situaciones aún peores. Así lo reafirma el informe de la Corporación RAND sobre las siete lecciones que arrojaron los conflictos en el mundo en las últimas dos décadas, en su primera lección dice: “... falta de comprensión y aplicación del arte estratégico..." (Linda Robinson, 2014, pág. 3).

Es por ello, que se realizará en este trabajo una aproximación a la necesidad que el nivel estratégico tiene, de ponderar el nivel operacional en toda actividad que se emprenda con el empleo de las Fuerzas Armadas. Esto nos lleva directamente a efectuar un análisis sintético de la relación con los niveles, especialmente entre el nivel estratégico y el operacional, agregado un párrafo especial a la relación dentro del nivel estratégico con los actores que lo componen.

\section{Relación entre el nivel estratégico y el operacional.}

La conducción estratégica nacional para arribar a las acciones tácticas dependerá de un nivel de la conducción muy importante que es "el nivel operacional u operativo", quien es el encargado de receptar las intenciones de aquél nivel y traducirlo en lineamientos operativos para las fuerzas a disposición de las operaciones, y en definitiva es el gerente general de la crisis.

Nos dice el MC 20-01 (2016): "El Nivel Estratégico Político y Militar en cabeza del Presidente con el asesoramiento del Ministro de Defensa y el Estado Mayor Conjunto de las Fuerzas Armadas, y el nivel operacional en cabeza del Comandante Operacional designado como responsable único ante el nivel estratégico" (pág. 24). Traduciendo gráficamente lo expresado sería lo siguiente: 


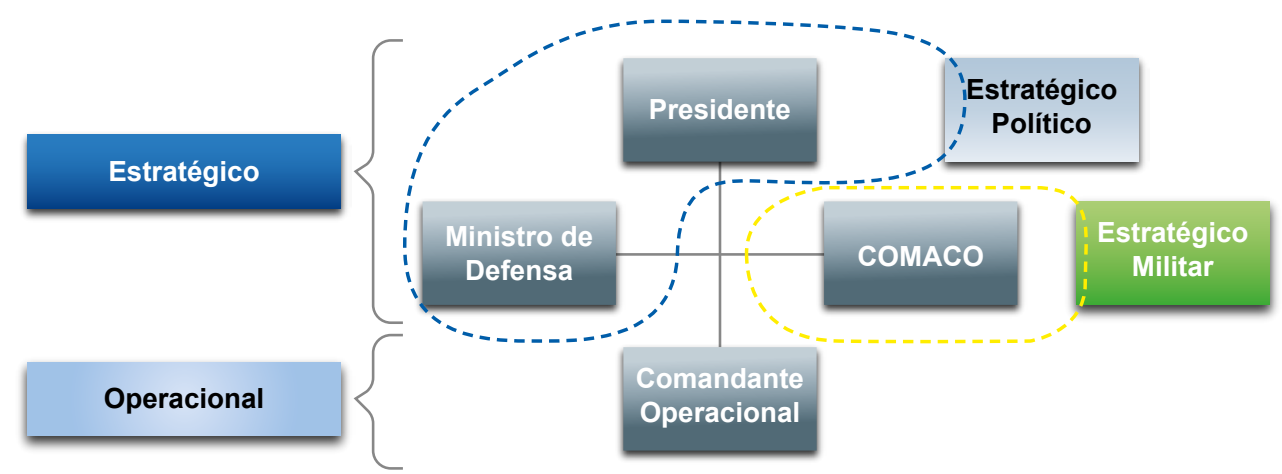

Figura 1. Relación jerárquica entre niveles de la conducción.

(Fuente: elaboración propia).

Se observa en el gráfico, la dependencia directa al Presidente del Comandante Operacional y la función de asesoramiento en el plano político del Ministro de Defensa, y en el plano militar la responsabilidad es del Comando Conjunto. Esto facilita la interpretación del poder político con el uso de las Fuerzas, y contribuye a que los órganos de dirección de las instituciones apoyen al Comandante único en la Zona de Crisis.

Con esta relación jerárquica, se robustece el nivel operacional, esto ha ocurrido desde la segunda guerra mundial hacia nuestros tiempos, ya no depende de la idiosincrasia o tradiciones de cada Estado, sino que esto es experiencia en la administración de crisis y en definitiva pericia al actuar. Este nivel no deberá ser soslayado, sino por el contrario reforzado convenientemente; porque la principal experiencia de los fracasos en la administración de las crisis, está dado porque este nivel no tuvo su real protagonismo.

A pesar de los nuevos escenarios y formas de combate, la finalidad de la guerra sigue concibiéndola como la continuación de la política por y con otros medios. Es por ello que su preocupación radica en cómo se vincularon los comandantes operacionales con los niveles estratégicos para hacer una correcta transferencia de sus intenciones a la táctica. (Locatelli, 2015, pág. 14)

¿Cuál sería la primera medida conveniente desde el nivel estratégico? La primera medida de relevancia sería el nombramiento de un comandante operacional en la zona de crisis, quien convenientemente reforzado con un estado mayor y fuerzas disponibles a tal fin, podrá iniciar la etapa quizás más importante para él, que es la comprensión de su misión impartida por el nivel estratégico a través de su estado final deseado con la comprensión del escenario operacional donde actuará (Estado Mayor Conjunto, 2016). A partir del inicio de esta etapa, será conveniente que las instituciones y organizaciones gubernamentales y de otra índole, se encuentren alertadas que quien lidera la actividad en la zona de crisis, es aquel comandante; será de fundamental importancia que el trabajo que desarrolle tenga características de inter-agencial y conjunto, y hasta a veces multinacional.

Seguramente el comandante de la zona de crisis deberá estar preparado para recibir presiones de todos los estamentos, pero debe ser apoyado por el Poder Ejecutivo y el nivel estratégico militar (En el Ecuador COMACO) para que su problema esté centrado sólo en las operaciones en desarrollo en dicha zona de crisis.

Para que todo se desvuelva con normalidad, deberá existir una cadena de responsabilidades en la producción de los insumos que circular entre los distintos niveles de la conducción, para que no haya interpretaciones erróneas y cada nivel pueda desarrollar con técnica su ámbito de responsabilidad para llevar a buen puerto la crisis.
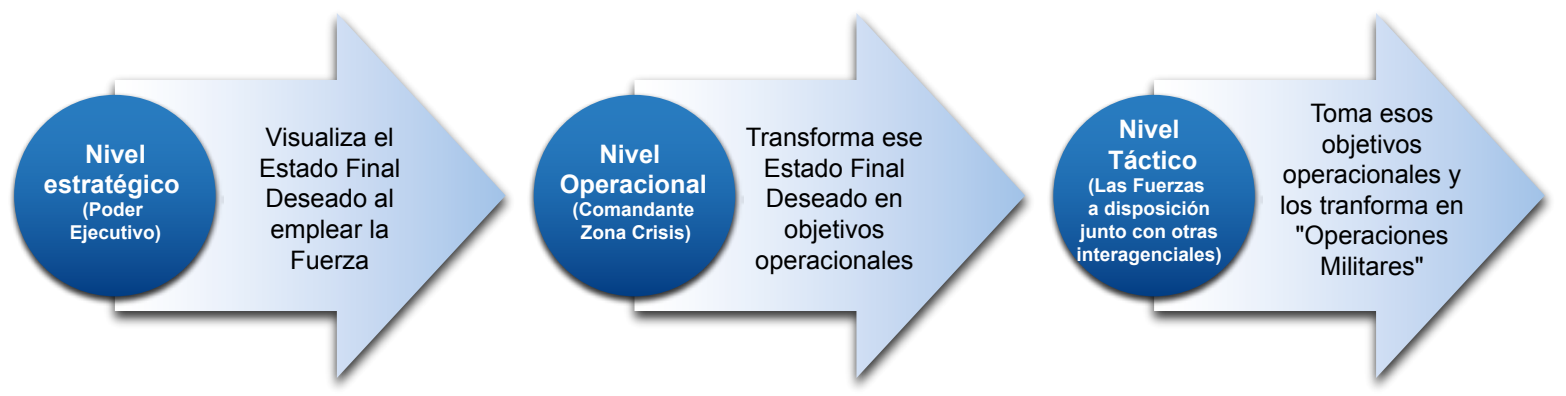

Figura 2. Insumos a producir para el nivel de conducción que le sigue.

(Fuente: elaboración propia). 
Parece simple, pero en reiterados episodios a lo largo de la historia occidental nos encontramos con comandantes operacionales que no disponen de estados mayores, o no tiene atribuciones necesarias en la administración de la crisis, o el nivel estratégico militar se confunde con el operacional, desconociendo uno de los principales axiomas o principio de la conducción en cualquier índole de las actividades militares que es la “unidad de comando". (Rattenbach, 1983)

El primer insumo elemental que debe producir el nivel estratégico es el Estado Final Deseado (en adelante EFD), todos los niveles de la conducción deberán disponer del suyo, como una cadena de objetivos el primer de los EFD debe ser convenientemente definido para que el resto de los niveles coadyuven a aquel.

Cada operación debe centrarse en un estado final claramente definido, decisivo y alcanzable, el éxito depende de la descripción precisa de esas condiciones, ya sean tangibles o intangibles, militares o no militares. Estas condiciones pueden orientarse a factores físicos o psicológicos. Pueden describir o relacionarse con percepciones, niveles de comprensión, cohesión entre grupos o relaciones entre organizaciones o individuos. Al final, forman la base para tomar decisiones las cuales garantizan que las operaciones avancen consistentemente hacia el estado final deseado. (Cardon, 2010, pág. 6)

Es así que la relación de estos niveles debe ser lo más cercana y precisa en cuanto a lo que se busca con el empleo de la fuerza, por ello ¿cómo reforzar entonces el nivel operacional?

\section{Reforzar el nivel operacional.}

Al hablar del nivel operacional nos invade la incertidumbre; primero, porque el nivel táctico es por excelencia nuestro lugar de confort, allí hay infinidad de procedimientos, están las cosas claras y precisas, no demanda esfuerzo en pensar fuera de ese encuadramiento, es en ese lugar donde nos sentimos seguros; segundo, el nivel estratégico es una suerte de "campo de ideas" donde es casi imposible que afecte en el aquí y ahora, porque las acciones o pensamiento en este nivel tendrán efectos allá lejos en el tiempo, entonces también es un lugar relativamente cómodo. Por ello, el nivel operacional para nosotros representa un terreno explorado pero poco conocido, donde las incertidumbres tienen efectos en el aquí y ahora, a diferencia del nivel estratégico que será un problema de otro, o en el táctico que busco la respuesta en el manual de procedimientos.

Este nivel, es un gran desafío para nuestros Generales y sus estados mayores, es el nivel que conecta la sabiduría política con el brazo fuerte armado, es el lugar donde ellos deberían de sentirse a gusto, pero ¿será tan así? ¿Hay posibilidades que se disponga de estados mayores o asesores que dominen este nivel, aportando la técnica necesaria al instinto del comandante?

Todo ello, siempre en situaciones de crisis, que no se resuelven anticipándose a constituir previamente comandos operacionales permanentes, o agregando a la fuerza un alto entrenamiento en procedimientos no militares, etc. Sino que, el adiestramiento y entrenamiento en el actuar del nivel operacional será el mejor aporte a la administración de estas crisis, es decir en la conducción por excelencia de las operaciones militares conjuntas, permitiendo así disponer de personal jerárquico entrenado en el abordaje de situaciones complejas y conflictivas, que normalmente escapa al entrenamiento primario de las Fuerzas Armadas.

Pues bien, ¿existe la posibilidad de lograr esa técnica para abordar los problemas complejos de mejor manera? A continuación la respuesta.

\section{Arte y Diseño Operacional: una herramienta necesaria.}

A lo largo de las experiencias en las distintas Fuerzas Armadas del mundo, que se pudo acceder, se observa la necesaria distinción entre los niveles de la guerra, del planeamiento y de la conducción, siempre argumentando que esa distinción es indispensable, porque se tratan problemas de distinta naturaleza. Dicha distinción en la práctica, no es tan palpable y hasta muchas veces la tomamos como ridículamente limitativa del accionar del comandante. Pero a la hora de la verdad, aquél borra los límites, y haciendo caso omiso de las experiencias en crisis pasadas, comete graves errores que muchas veces radican en la ansiedad que provoca la espera de un asesoramiento o el propio procedimiento de toma de decisiones, que seguramente será distinto a lo que se espera, o la persona a cargo no procederá como lo espera aquél, y así nuevamente el instinto le gana a la técnica.

Nos dice el Manual de Campaña 5-0 (2010) del Ejército de los Estados Unidos de América: "El diseño no es un proceso ni una lista de control. Es una metodología de pensamiento crítico y creativo para ayudar a los comandantes a comprender el ambiente, analizar problemas y considerar posibles planteamientos que puedan beneficiarse de las oportunidades, que identifiquen vulnerabilidades y prevean las transiciones durante una campaña.” (pág. 7)

La necesidad de técnicas más adecuadas al nivel operacional, donde la propia naturaleza de los problemas son distintos que en lo táctico o lo estratégico, hicieron surgir esta forma de abordaje, que sus primeros pasos podremos ubicarlo en Rusia de posguerra 1923 con el término "operativnoe iskusstvo", "operational art" o "arte operacional", propuesta del General 
Alexander Andreyevich Svechin en conferencias brindadas en la Academia de Guerra de entonces, cayendo en desuso por la purga efectuada en las Fuerzas Armadas rusas ordenada por Stalin (Blythe, 2018). Luego en la década del 70 en el siglo pasado también fue adoptada por el Ejército de los Estados Unidos de América, hoy ya en la OTAN, en Argentina, Brasil, etc.

El "arte y diseño operacional" viene a dotar a esos intuitivos comandantes de la suficiente pericia técnica para abordar los problemas complejos, sin eliminar su instinto sino todo lo contrario potenciarlo. Donde su formación en el tema les otorgará una gran ventaja a la hora de la "espera" en el asesoramiento, y lo que es más importante las herramientas necesarias para comprender el problema y poder identificar los posibles abordajes a priori del asesoramiento conveniente, y de esta manera orientar convenientemente a sus estados mayores.

El diseño proporciona las herramientas cognitivas necesarias para reconocer y gestionar transiciones, identificar y emplear soluciones adaptables e innovadoras, crear y aprovechar oportunidades, proteger potenciales vulnerabilidades y gestionar riesgos a su favor durante estas transiciones. (Cardon, 2010, pág. 12)

Entonces, al formar a nuestros comandantes y asesores en estas herramientas del "arte y diseño operacional" podremos estar seguros que conocen la técnica que el mundo ha desarrollado para afrontar problemas complejos, donde las posibles soluciones no son sólo de índole bélica sino de otros aspectos también.

La primera pregunta que podría surgir en este tema es: ¿Dónde está escrita la herramienta para poder acceder a la misma y de esta forma estudiarla? La respuesta no es del todo alentadora para las Fuerzas Armadas del Ecuador, porque no se dispone de un plexo dogmático para poder acceder a la misma.

En el caso de la República Argentina, dispone de un reglamento denominado MC 20-01 "Planeamiento para la acción militar conjunta - nivel operacional" que aglutina en el aspecto conjunto la doctrina de "arte y diseño operacional", además de ello existe una publicación de la Escuela Superior de Guerra Conjunta, un texto didáctico para uso académico que se titula "Arte y Diseño Operacional" escrito por el Almirante (SP) Alex Kenny, el Coronel (SP) Omar Locatelli y el Coronel Leonardo Zarza, dos de ellos estuvieron en el país dando conferencias sobre temas afines. Para el caso de la OTAN, existe un reglamento de nivel operacional que es el "Comprehensive Operations Planning Directive" (COPD). Para los EUA existe en el nivel operacional el Joint Publication (JP) 5-0 del 16 de Junio de 2017.

\section{Elementos del Diseño Operacional:}

Para elaborar un diseño operacional es necesario el correcto empleo de los elementos del diseño, para ello se realizará una breve descripción de los mismos y una reflexión sobre la importancia a la hora de elaborar las órdenes para el nivel táctico.

5.1. Estado final deseado: Según el PC 20-01 (2017) “El Estado Final Deseado es la situación política y/o militar que debe existir cuando la operación se dé por terminada en términos favorables. Se considera un estado final para cada uno de los niveles: estratégico nacional, estratégico militar y operacional.” (pág. 18)

Podemos observar que el Poder Ejecutivo Nacional junto a su órgano de asesoramiento deberán imaginar cómo quedará el escenario luego del empleo de las Fuerzas Armadas, para ello deberá auxiliarse de quienes técnicamente son capaces de elaborar escenarios prospectivos, además de los asesores en el área de defensa y militar, porque la decisión de emplear estas fuerzas no puede tomarse sin los análisis previos convenientemente elaborados.

El desarrollo de este trabajo los deberá llevar a preguntarse si con los medios disponibles se podrá cumplir el fin que se busca, sin lugar a dudas el esquema "Fines, Modos y Medios" junto con el "Riesgo" tiene un papel muy importante en esta etapa. Donde los "Fines" se plantean a través de este "Estado Final Deseado estratégico" (en adelante EFDE), es un arduo trabajo para llegar a consolidar en una breve oración la situación deseada al finalizar el empleo de la fuerza, es así que aquella oración deberá tener las características de contundente, abarcativa y reflexiva.

Existen argumentos a favor de varios autores sobre las necesidades de elaborar EFDE "político" y otro EFDE "militar", pero para quien suscribe deberá ser necesario distinguir entre un planeamiento premeditado (deliberado) y otro de crisis porque éste sólo le será útil disponer del EFDE político para que con su Comando y Estado Mayor pueda elaborar el siguiente estado final que es el operacional, quedando para el planeamiento con mucho más tiempo el EFDE "militar", porque el consumo que puede darse a la duplicidad de estados finales puede ser un gasto innecesario de tiempo en planeamiento de crisis. Entonces, el EFDE político debería ser suficiente para el siguiente eslabón de comando que es el Comandante Operacional. 


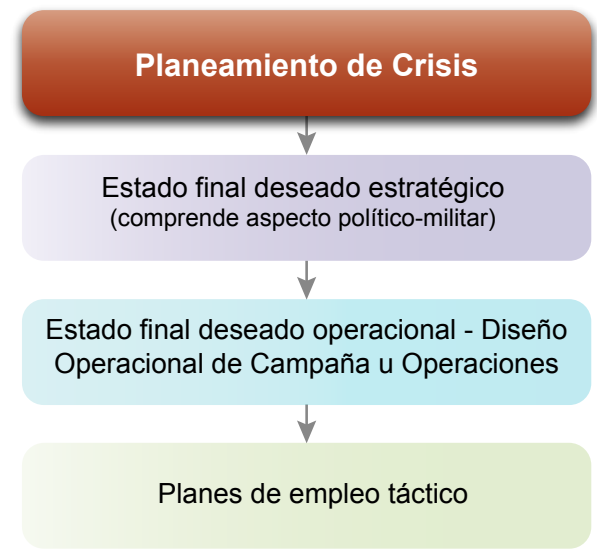

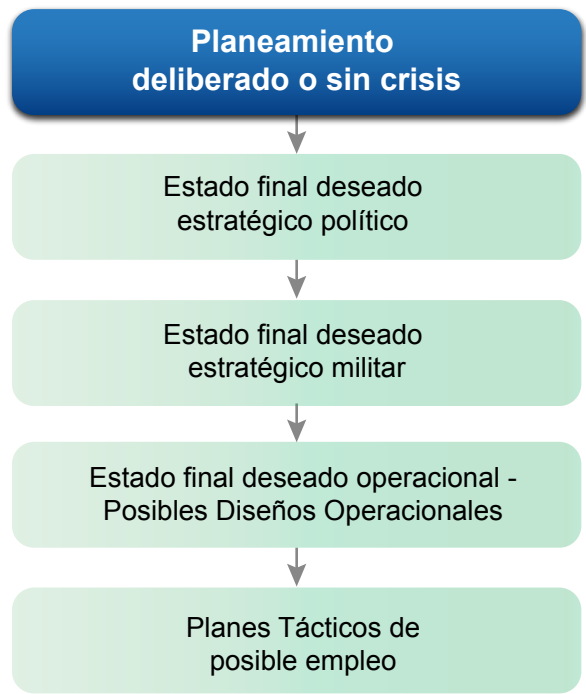

Figura 3. Encadenamiento de los estados finales con crisis y sin crisis.

(Fuenta: elaboración propia).

Podemos observar en el gráfico dos columnas, una referida al planeamiento ante una crisis y la otra sin ella. En la columna de la izquierda, se muestran los tres niveles de la conducción y para quien suscribe, del planeamiento ante una situación de crisis, con sus correspondientes actividades relacionada con el resto de los niveles, comenzando por el primer nivel (estratégico) el producto a elaborar debe ser uno sólo (político-militar juntos), porque considero no será bueno duplicar los esfuerzos en este nivel sino por el contrario debe apoyar al nivel operacional. En la columna de la derecha, se observan los niveles de planeamiento en tiempos de paz, allí se coloca el nivel estratégico militar con su producto, porque no se tiene la premura del actuar ante una crisis, lo que no significa que no sea para actuar sobre una amenaza real y actual, pero ante la crisis se puede prescindir de su planeamiento, no así de su asesoramiento conforme se lo establezca. Esto es reafirmar lo expresado supra sobre la necesidad de reforzar el protagonismo del nivel operacional.

Este elemento del diseño es el fundante de todo el sistema, nos dice Barrales (2014) respecto a la existencia de estados finales de máxima y mínima:

Asimismo, en los niveles Estratégico y Estratégico Militar se establece un estado final "de máxima" y otro "de mínima". El EFD "de máxima" del atacante corresponde a su mayor aspiración de "adquisición"; y para el caso del defensor expresa su máxima expectativa de "preservación". Por su parte, el EFD “de mínima" para el atacante significa no haber obtenido todo lo que quería pero sí más de lo que tenía antes de iniciar el conflicto, manteniendo con lo ganado un adecuado poder de negociación, y todo ello a un costo aceptable; y para el defensor implica conservar al final del conflicto lo razonablemente aceptable, de acuerdo a la relación de fuerzas de las partes enfrentadas. (pág. 94)
Finalmente cabe agregar que el Estado final deseado es el elemento del diseño más importante, donde todas las fuerzas y energías apuntan a alcanzarlo, por ello la pericia que debe tener el nivel estratégico para producirlo es de innegable relevancia.

5.2. Objetivo operacional: en íntima relación con el estado final deseado operacional fue definido por el PC 20-01 (2017) de la siguiente manera.

Para lograr el Estado Final Operacional deberán alcanzarse una serie de objetivos, (secuencial o simultáneamente) denominados "relación de objetivos". Se denomina Objetivo Operacional (OO) al último de la dicha relación de objetivos mencionada, porque finalmente concreta el Estado Final Operacional en ambiente operacional. Los anteriores, en dicha relación, son de nominados objetivos intermedios (OI). También puede coexistir un Objetivo Operacional Principal (OOP) con uno o varios Objetivos Operacionales Secundarios (OOS), donde el principal es alcanzado por el esfuerzo principal y los demás por los respectivos esfuerzos secundarios y de apoyo. Los objetivos del Nivel Operacional pueden ser de distinta naturaleza (material o inmaterial). Deben ser claramente definidos y comprendidos por todos. (pág. 18)

Este será quizás el producto más importante del Comandante Operacional luego del Estado Final Deseado, porque a través de ellos/el materializará con las fuerzas el fin de la campaña u operaciones.

Debe tener las características de todos los objetivos: obtenible - identificable - alcanzable, siempre siguiendo la modalidad de redacción de objetivos (Acción + Objeto) donde la acción puede ser "proteger, obtener, conquistar, etc" y el objeto puede ser material o inmaterial, por ejemplo proteger una zona determina (material) o conquistar la voluntad de lucha (inmaterial). 
Este elemento del diseño, debe ser analizado convenientemente porque se transforma en un elemento fundante, donde las líneas de operaciones se conformarán apuntando hacia ese objetivo y por sobre todas las cosas será el origen del centro de gravedad.

5.3. Centro de gravedad; este elemento del diseño operacional se debería de definir de la siguiente forma: núcleo de poder sistémico que tiene la capacidad de alcanzar el objetivo impuesto, en su nivel de la conducción considerado, en un tiempo y espacio determinado. Esta definición nos permite concentrar tanto un "ente primario" como "fuentes de poder" que son las dos denominaciones que lideran las definiciones del centro de gravedad en la actualidad.

Aquí nos vamos a encontrar con uno de los elementos que tienen mayor desarrollo doctrinario en cuanto a su evolución y estado del arte, así nos dice Gniesko (2017)

El centro de gravedad (CDG) es un término que se asocia a un concepto táctico, cuyo significado está relacionado a la concentración de esfuerzos o centros de enfoques, pero que en la actualidad en la doctrina militar tiene otro significado más, al incorporarse una nueva acepción. Esta última acepción, va más allá de un empleo táctico, que convierte al CDG en una preocupación central de todo comandante, otorgando una herramienta analítica de determinación del mismo, cuya fuente del término ha sido el desarrollo alcanzado en los Estados Unidos de América (EUA). (pág. 1)

Existe una matriz desarrollada por Gniesko (2019) que puede ayudar a comprender el encadenamiento de los elementos del diseño, especialmente los necesarios para elaborar un diseño operacional.

\subsection{Líneas de operaciones: Según el PC 20-01 (2017).}

Las LDO son aquellos conjuntos de acciones relacionadas entre sí, normalmente dependientes unas de las otras, cuya ejecución permitirá ir alcanzando los PD, que a su vez nos darán acceso al CDG. Los Comandantes las emplean para dirigir la capacidad de combate hacia un fin deseado, aplicándolas en las tres dimensiones del espacio, para converger y neutralizar/dislocar/batir al o los CDG de las fuerzas adversarias. Su principal utilidad, a los efectos prácticos del planeamiento de las operaciones, se encuentra en el hecho de que se trata de herramientas que sirven para sincronizar, orientar y concentrar - tanto en tiempo como en espacio- los esfuerzos, la potencia de combate $y$, en definitiva, los efectos de las diferentes fuerzas y mandos componentes de la operación, sobre un mismo objetivo. (pág. 25)

Es un elemento utilizado a lo largo de la historia para conectar las ciudades con su Ejército, así como las fuerzas con sus objetivos. Cómo vamos recorriendo hasta ahora, son términos con cierta familiaridad y utilizados desde la táctica a la estrategia, el tema aquí es el significado y su combinación.

\subsection{Puntos decisivos: Según el PC 20-01 (2017)}

Son un conjunto de condiciones o sucesos clave (coordinados en el tiempo y el espacio), tanto para el oponente, propia fuerza o medio ambiente, que deben ser alcanzados a través de acciones y efectos que exploten las VC y que permitan neutralizar un CDG. El cumplimiento de los PD debe ser mensurable. Lo más importante es establecer claramente el propósito de cada PD con el efecto buscado sobre el CDG. (pág. 23)

Estas condiciones o sucesos deben ser los eslabones que unen las líneas de operaciones desde las primeras acciones hasta la conquista de los objetivos operacionales (Principal y Secundarios).

El arte operacional deberá estar refinando en la concepción de estas situaciones que tendrán que ser como notas en una melodía, mientras más precisos sea la búsqueda de sus efectos mayor, será la eficacia de los mismos.

\subsection{Momento (Momentum): Según el PC 20-01 (2017)}

Es la oportunidad de ejecutar una acción que permita explotar las vulnerabilidades del oponente. Crear el momento es concebir el diseño operacional de manera tal de lograr el efecto deseado sobre el CDG del oponente rápidamente. El momento permite a los Comandantes crear oportunidades, para enfrentar al oponente desde direcciones y/o con capacidades inesperadas. Todos los niveles de la conducción del conflicto pueden contribuir al logro del momento del nivel operacional. Será responsabilidad del Comandante Operacional de asesorar a la Estrategia Militar y Nacional sobre las eventuales necesidades o requerimientos para alcanzar el momento operacional según su diseño operacional y así, no desperdiciar oportunidades. (pág. 24)

El presente elemento del diseño otorga la posibilidad de administrar tiempos, pausas y esfuerzos, porque la presión constante reciente cualquier estructura, por ello este elemento va a permitir conservar poder de combate y explotar vulnerabilidades cuándo las mismas sean palpables en el adversario.

\subsection{Ritmo: Según el PC 20-01 (2017).}

Muy ligado con el anterior nos permitirá mantener la suficiente presión sin resentir la organización o estructura.

En el diseño operacional es necesario tener en cuenta la adecuada aplicación del ritmo la cual significa 
mantener la presión constante sobre el oponente disminuyéndole su capacidad de respuesta. El ritmo tiene significado militar sólo en términos relativos. Cuando un ritmo propio sostenido supera la capacidad del oponente para reaccionar, las fuerzas propias pueden mantener la iniciativa y obtener una ventaja marcada y generando con ello una adecuada libertad de acción. Controlar y/o alterar ese ritmo es necesario para retener la iniciativa. Para maximizar las propias capacidades puede ser necesario ajustar el ritmo de la operación. (pág. 24)

\subsection{Maniobra operacional: Según el PC 20-01 (2017)}

La Maniobra Operacional (MO). Es la combinación de esfuerzos operacionales, a ser llevados a cabo mediante el mejor empleo de los recursos y fuerzas disponibles, en un tiempo y espacio dados, para alcanzar el Objetivo Operacional (OO). Las dimensiones de la MO son el espacio, el tiempo y la masa. Una MO puede ser ofensiva o defensiva, dependiendo de la actitud del esfuerzo operacional principal, que es quien lo determina.

Este elemento nos permitirá realizar una aproximación adecuada a las líneas de operaciones y la conexión con los objetivos operacionales principales, porque nos hace visualizar los posibles esfuerzos operacionales a llevar a cabo, a veces se soslaya porque no se le encuentra finalidad, pero cuando se la conecta con el $\mathrm{Ob}$ jetivo Principal y la agrupación de líneas de operaciones se verá claramente cuál es su finalidad.

\subsection{Esfuerzo Operacional: Según el PC 20-01 (2017)}

El esfuerzo operacional es la aplicación y/o concentración de medios, fuerzas o efectos en un área y oportunidad particular, donde un Comandante busca obtener resultados favorables. Los esfuerzos operacionales se materializan a través de una o varias líneas de operaciones y pueden cambiar en cada fase o momento de la Campaña. Los esfuerzos operacionales pueden ser principales o secundarios. En cada MO, habrá siempre un solo esfuerzo operacional principal y ninguno, uno o varios esfuerzos operacionales secundarios. No deben enunciarse por separado, sino en conjunto.

Cómo lo define el reglamento en cuestión, siempre tiene que ir de la mano de la maniobra operacional, porque estamos en presencia del género y la especie del direccionamiento hacia los objetivos, los esfuerzos hacia los secundarios y la maniobra hacia el principal, pero siempre conectados. Cuando se visualicen los esfuerzos en la maniobra, comenzarán a surgir las posibles pausas operacionales o los alcances, siempre observando la necesidad de no llegar al punto culminante y obligar al adversario a que él lo alcance. Veremos además, como se conectan los requerimientos críticos del centro de gravedad del adversario con estos esfuer- zos, y además como comienzan a aparecer los puntos decisivos, que son la explotación de vulnerabilidades críticas del centro de gravedad. Y así las relaciones que se expresaron debe ser para cada elemento del diseño operacional.

\subsection{Punto culminante: Según el PC 20-01 (2017)}

Es la situación en el desarrollo de un conflicto, en la cual la relación de poder entre los actores o fuerzas, impide a uno de ellos mantener la actitud en curso con razonable expectativa de éxito, obligándole a adoptar un cambio de la misma o establecer una pausa operacional. El punto culminante es un concepto aplicable en los tres niveles del conflicto. El arte reside en alcanzar los objetivos de una operación antes del punto de culminación de las fuerzas propias.

Ya mencionada la relación con el resto de los elementos del diseño tenemos definidos casi todos los más importantes, a ellos pueden agregarse otros para mejorar la visualización y comprensión, pero siempre tratando de ser simples en esta elaboración que deberá de poder ser transmitida a los comandos subordinados.

\section{Proceso de planeamiento y su actualización:}

A medida que se siga avanzando en la comprensión y utilización de las bondades que nos otorga el arte operacional, posiblemente deberá de repensarse los procesos de toma de decisiones que se disponen en las Fuerzas Armadas. Porque existirán análisis sobre abundantes e innecesarios que el proceso clásico deberá ser obviado.

Por ello, hay que buscar integrar el arte y diseño operacional como parte del proceso de la toma de decisiones, teniendo presente no reducirlo a mecanismos lineales, contemplando la necesidad de conservarlo con sus principios intactos, pero con una transición adecuada hacia los clásicos procesos de toma de decisiones, de manera tal que no haya desconexiones entre ellos.

\section{Conclusiones:}

Nobleza obliga, la primera de las conclusiones a las que se debe arribar es que las Fuerzas Armadas del Ecuador tiene que reforzar el nivel operacional, no sólo con organizaciones, sino con aspectos doctrinarios, operativos y conceptuales en su personal superior. Ante ello, la Academia de Guerra de la Fuerza Terrestre tiene como desafío un proyecto de manual de "Arte y Diseño Operacional".

Sin lugar a dudas la información es un factor relevante para el actuar de las Fuerzas Armadas, nos dice Kaplan (2015): "Como factor productivo, la información se vuelve tanto o más importante que el capital, el trabajo y la tierra (...) produce cambios en todos los niveles y aspectos de la producción y el comercio, na- 
cionales y mundiales, en sí misma y en combinación con otras ciencias, técnicas y producciones." (pág. 55) Por ello, se debe repensar donde se impartirán estos conocimientos y como se articularán para que no sean de una fuerza sino para las tres de manera conjunta.

Junto a esto, se debe disponer de organizaciones abiertas, donde la relación de colaboración es un valor a crear y mantener, nos aporta a esto Cardon (2010) "El empleo con éxito del diseño depende del liderazgo eficaz y decisivo que yace fundamentalmente en la participación activa y diálogo y colaboración continua..." (pág. 15)

Por último, el valor más importante que tiene este tema es que trabajo se centra en "el hombre y su pensamiento", quien encontrará respuestas a través de herramientas que generan creatividad y pensamiento crítico, promoviendo la iniciativa. Observamos que dentro de las recomendaciones de la Corporación RAND sobre los últimos conflictos nos dice Linda Robinson (2014):
El personal innovador y multifuncional puede hacer que una fuerza más pequeña sea más efectiva, pero los incentivos deben ser sistémicos para recompensar al personal por la creatividad, la asunción de riesgos y la adquisición de múltiples especialidades. El principio del comando de la misión podría profundizarse para permitir una mayor descentralización y delegación de iniciativa.

Sin lugar a dudas, se está a tiempo de lograr el cambio orgánico y dogmático necesario para transformar la fuerza, utilizando elementos que aporten eficiencia a la hora de actuar, pero se deberá salir del confort que otorga lo conocido y adentrarse en el esfuerzo intelectual que produce cambios valederos y perdurables.
[1] Barrales, J. P. (2014). Punto Culminante y Estado Final Deseado. Boletín Centro Naval de la República Argentina. Obtenido de https:// www.centronaval.org.ar/boletin BCN835/835-BARRALES.pdf

[2] Blythe, W. (Diciembre-Noviembre de 2018). Militar Review. Obtenido de https://www.armyupress.army. mil/Journals/Military-Review/ English-Edition-Archives/NovemberDecember-2018/Blythe-OperationalArt/

[3] Cardon, E. (2010). La planificación y el arte del mando de batalla . Military Review, 10-21.

[4] Estado Mayor Conjunto, F. A. (2016). MC 20-01.

[5] Exum, A. (julio de 2017). The Atlantic. Obtenido de https:// www.theatlantic.com/politics/ archive $/ 2017 / 07 /$ the-danger-ofturning-the-us-military-into-apolitical-actor/534624/

\section{REFERENCIAS}

[6] Frank, H. (2009). Hibrid Warfare and Challenges. Joint Forces Quarterly. Obtenido de http://smallwarsjournal. com/documents/jfhoffman.pdf

[7] Gniesko, C. I. (2017). Evolución y estado del arte del centro de gravedad en los Estados Unidos de América. Military Review, pp.1-8.

[8] Kaplan, M. (2015). Las crisis del estado nacional latinoamericano. Obtenido de 7: https://www7.tau. ac.il/ojs/index.php/eial/article/ view/1301/1326

[9] Kipp, J. (s.f.). "Doctrina militar soviética y los orígenes del arte operacional, 1917-1936”. Obtenido de http://www.dtic.mil/dtic/tr/fulltext/ u2/a195053.pdf

[10] Kissinger, H. (Agosto de 2019). The Atlantic. Obtenido de https:// www.theatlantic.com/magazine/ archive/2019/08/henry-kissinger-themetamorphosis-ai/592771/
[11] Linda Robinson, P. D. (2014). RAND Corporation. Obtenido de Mejora de la competencia estratégica - RR816-A: https://www.rand.org/pubs/ research_reports/RR816.html

[12] Locatelli, O. (2015). Los Generales y los Elementos del Diseño Operacional . Visión Conjunta, 31-36.

[13] Mariano, B. (Julio-Diciembre de 2017). Universidad Militar Nueva Granada. Obtenido de https://www. redalyc.org/pdf/927/92751348003.pdf

[14] Rattenbach, B. (agosto de 1983). Casa Rosada. Obtenido de https:// www.casarosada.gob.ar/informacion/ archivo/25773-informe-rattenbach

[15] Rosa, B. (2016). Cambrige.org. Obtenido de https://www.cambridge. org/core/journals/americanjournal-of-international-law/article/ how-everything-became-war-andthe-military-became-everything-byrosa-brooks-new-york-new-yorksimon-schuster- 2016-pp-viii-438index-2995 /D3595995CD92844 3E23356BBB3310B0D

[16] US, A. (2010). Manual de Campaña 5-0. Ejército de los Estados Unidos de América. 